\title{
Immunological findings in relatives of patients with rheumatoid arthritis
}

\author{
Madalina-Pusa Duna', Denisa Predeteanu', Florian Berghea ${ }^{1,2}$, \\ Daniela Opris-Belinski ${ }^{1,2}$, Andra Balanescu ${ }^{1,2}$, Violeta Bojinca ${ }^{1,2}$, Ruxandra Ionescu ${ }^{1,2}$ \\ ${ }^{1}$ Department of Internal Medicine and Rheumatology, "Sf. Maria” Clinical Hospital, Bucharest, Romania \\ 2"Carol Davila" University of Medicine and Pharmacy, Bucharest, Romania
}

\begin{abstract}
Rheumatoid arthritis (RA) is a chronic and systemic inflammatory disease, with a very complex genetic component, being the most common rheumatic inflammatory disease. Family aggregation and the presence of autoantibodies in patients with RA suggest that the disease has autoimmune pathogenesis, despite the fact that the etiology is not fully known. The family members of patients with rheumatoid arthritis are at increased risk of developing RA and are potential candidates for predictive testing. Identifying relatives at risk of RA may allow targeted interventions to reduce the risk and catastrophic consequence of future disease.
\end{abstract}

Keywords: rheumatoid arthritis, autoantibody, autoimmunity, relatives

\section{INTRODUCTION}

It is well known that RA is a complex disease and the susceptibility and severity of the disease are probably influenced by the combination of genetic and environmental factors. Environmental factors involved in RA include obesity, diet, smoking and a large number of infectious agents, including parvoviruses, proteins and various retroviruses. Genetic factors contribute significantly to the risk of RA (1). For seropositive RA, at least half of the risk is conferred by genetic risk factors. Population-based epidemiological studies have shown that having a family history of RA increases the risk of RA by approximately three to five times with the risk being higher in first-degree relatives than second-degree relatives (2).

Indeed, a number of ongoing prospective studies are recruiting the first-degree relatives of patients with RA to study disease mechanisms driving the switch to RA, to develop predictive algorithms for RA and to test interventions to reduce RA risk (3). While considerable research effort is thus focused on the first-degree relatives of patients with RA, and a qualitative study has gathered data relating to their views of preventive strategies, little is known about how such individuals view issues related to their susceptibility to and risk of developing RA, and how willing they would be to be assessed and tested to have this risk quantified $(4,5,6)$.

Identification of RA in relatives at initial presentation and treatment at earlier stage can affect disease course, prevent the development of joint erosions or retard progression of erosive disease. Early diagnosis and treatment may affect disease outcomes even to a remission state (7).

Smolik and co-workers demonstrated that first-degree relatives of patients with RA were significantly more likely to report experiencing joint symptoms compared to the control group. These relatives also exhibited a significantly higher prevalence of RA autoantibodies. There were modest trends for joint symptoms to associate with RA autoantibodies, and individuals who were both anti-CCP positive and RF-positive had the highest prevalence of joint symptoms (8).

\section{OBJECTIVE}

The main objective of this study is to evaluate the usefulness of serum biomarkers in specifying the risk of developing RA in relatives of patients with 
RA. A secondary aim was to correlate these results with disease activity, disease duration and other parameters of patients with RA.

\section{MATERIAL AND METHOD}

We performed a longitudinal, prospective study that was conducted over a nine months period (January 2019 - September 2019), which included 117 patients with rheumatoid arthritis. All patients met the ACR \& EULAR classification criteria of 2010 (9).

Also, eligible participants were the first and second-degree relatives of people with RA. 117 relatives were included. Participants were required to be at least 18 years of age and without a diagnosis of inflammatory joint disease. They were recruited at the tertiary-referral Clinic of Internal Medicine and Rheumatology of the "Sfânta Maria" Hospital in Bucharest. The relatives were tested for anticitrullinated protein antibodies (anti-CCP) and rheumatoid factor (RF). These immunological tests were performed according to the manufacturer's instructions.

\section{Data management and analysis}

We analysed the data applying Student's t-test, Chi-Square Test, ANOVA, and Pearson's correlation.

\section{Ethical considerations}

The study was carried out after approval from the hospital ethics committee. Patients were included in the study after being explained to the purpose of the study and giving a signed informed consent. Participation in the study was voluntary and patients were at liberty to withdraw from the study without any prejudice.

\section{RESULTS}

Multiple variables per patient were collected. Variables were divided into several groups:

1. Demographic data: age, gender and geographic region.

2. The extra-articular manifestations in patients with RA.

3. The assessment of the rheumatoid arthritis activity using DAS28 score and HAQ score in patients with RA.

4. The determination of anticitrullinated protein antibodies (anti-CCP) and rheumatoid factor $(\mathrm{RF})$ in relatives.

The present study included 117 patients with seropositive RA. The mean age of patients classified as RA was $59 \pm 13.6$ years.

From the results, it can be seen that the majority of the study population were female $(69 \%)$.

The evaluation of the patients in the group according to the duration of the disease showed that the average duration of the disease was $11 \pm 5.8$ years.

It is well known that in RA, the assessment of the activity of the disease is made based on composite indices that take into account both clinical and bio-

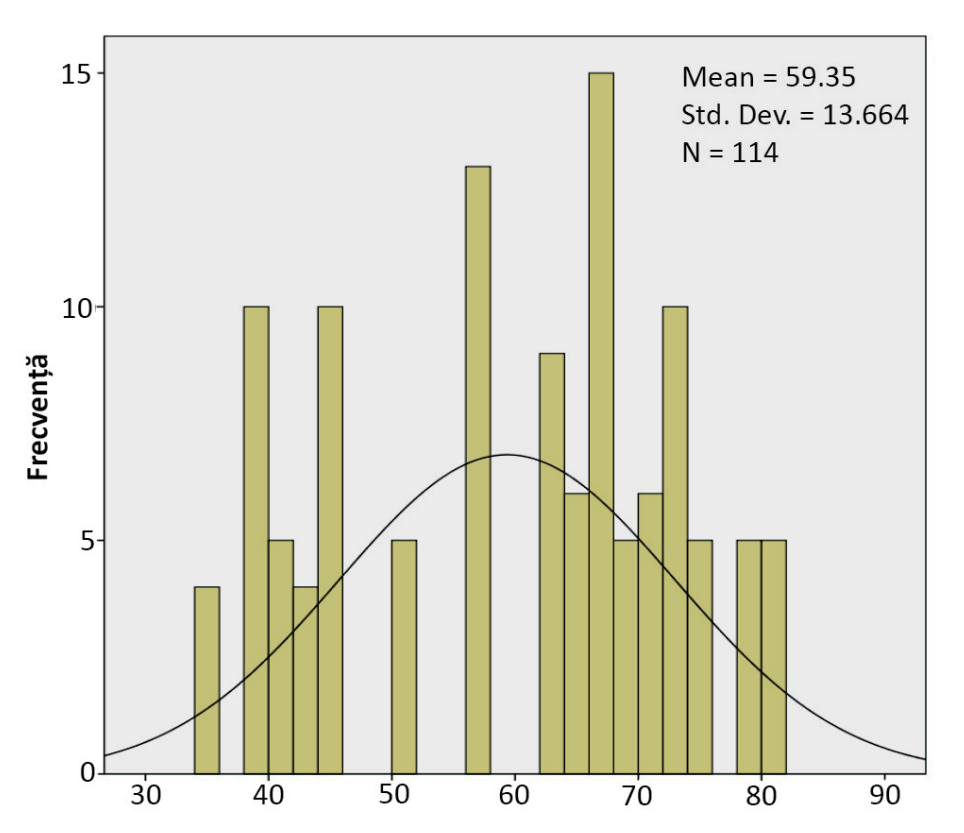

FIGURE 1. Mean age of patients with $A R$ 


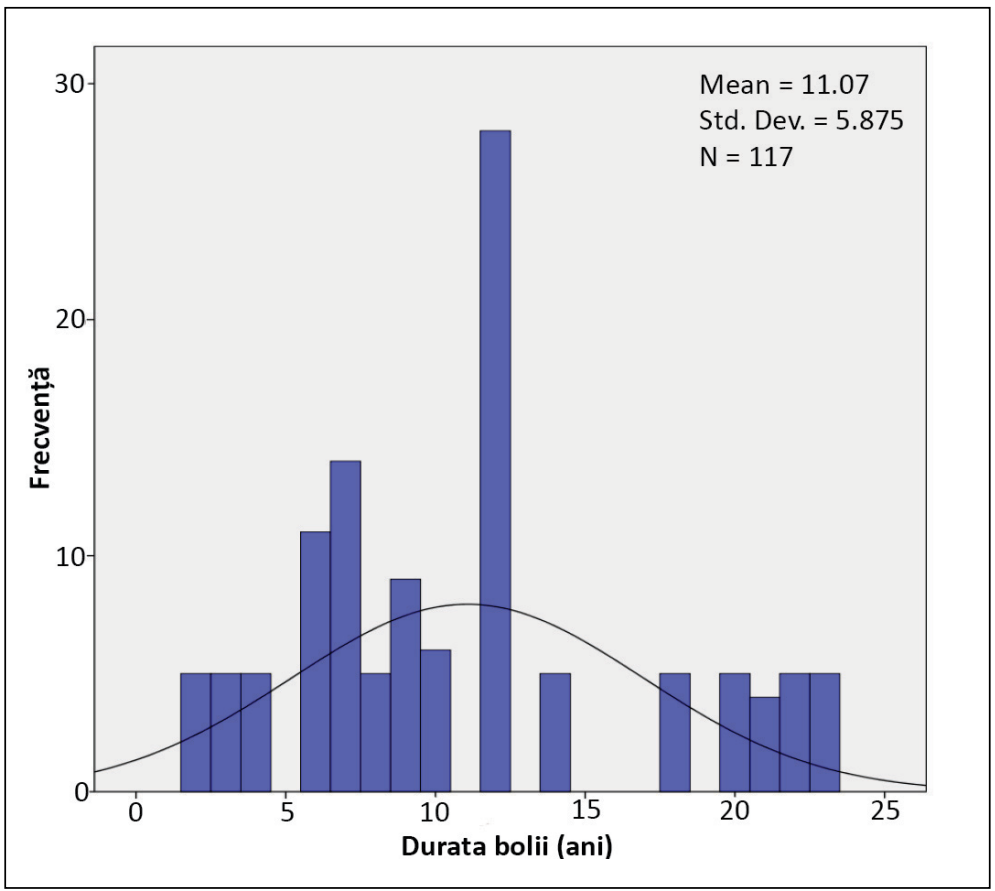

FIGURE 2. Mean duration of AR disease

logical parameters, such as the DAS28 score. The average value of the disease activity score was $3.3 \pm$ 0.8 with the limits between 2.1 and 6 .

The mean (interquartile range deviation) HAQ (health assessment questionnaire) in these patients was $1.12 \pm 0.41$, with a minimum of 0.3 and a maximum of 1.8 , meaning a moderate alteration in the quality of life of these patients. Furthermore, in the group of patients with RA, HAQ score tends to increase as the disease progresses but without statisti- cal significance $(\mathrm{p}=0.0935)$. However, there was a directly proportional correlation between HAQ and patient age $(\mathrm{r}=0.2, \mathrm{p}=0.02)$.

The present study also included also the evaluation of extra-articular manifestations in patients with RA. 17 patients had skin manifestations, 16 patients had cardiac manifestations, 15 had pulmonary manifestations, 11 patients had rheumatoid nodules, and 26 patients had mucosal disease such as Sjögren's syndrome.

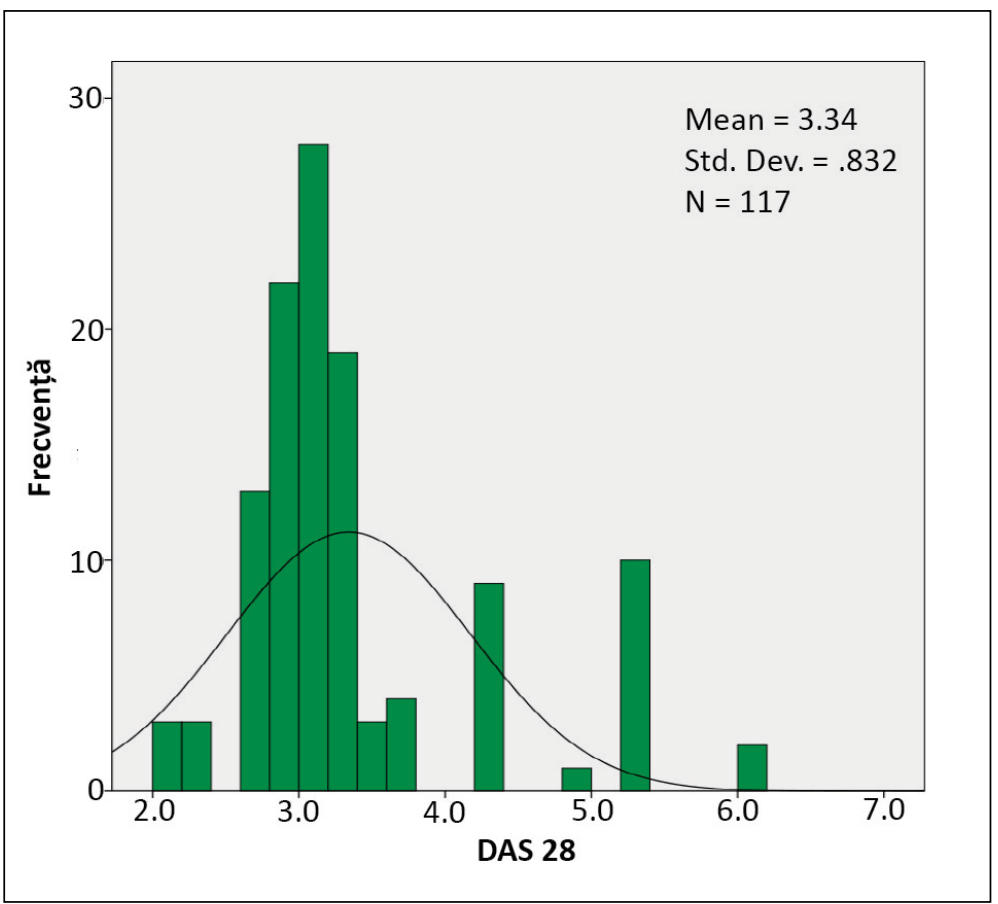

FIGURE 3. Mean values of DAS28 in RA group 
TABLE 1. Demographic data and disease characteristics in patients with $A R$

\begin{tabular}{|c|c|}
\hline Variables & $\begin{array}{c}\text { Mean value } \pm \text { standard } \\
\text { deviation (minimum; } \\
\text { maximum) or percentage } \\
\text { number (\%) }\end{array}$ \\
\hline Age (years) & $59 \pm 13.6(35 ; 81)$ \\
\hline Female & $81(69 \%)$ \\
\hline \multicolumn{2}{|l|}{ Geographic region } \\
\hline Rural areas & $63(53.8 \%)$ \\
\hline Urban areas & $54(46.1 \%)$ \\
\hline Mean disease (ys) & $11 \pm 5.8$ \\
\hline Smoking & $53(45.2 \%)$ \\
\hline \multicolumn{2}{|l|}{ Steinbrocker classification } \\
\hline I & $4(3.4 \%)$ \\
\hline II & $80(68.3 \%)$ \\
\hline III & $24(20.5 \%)$ \\
\hline IV & $9(7.6 \%)$ \\
\hline DAS28 (mean value) & $3.3 \pm 0.8$ \\
\hline HAQ (mean value) & $1.12 \pm 0.41$ \\
\hline \multicolumn{2}{|c|}{ Extra-articular manifestations } \\
\hline Skin & $17(14.5 \%)$ \\
\hline Rheumatoid nodules & $11(9.4 \%)$ \\
\hline Sjögren's syndrome & $2622.2 \%)$ \\
\hline Cardiac & $16(13.6 \%)$ \\
\hline Pulmonary & $15(12.8 \%)$ \\
\hline \multicolumn{2}{|l|}{ Treatment } \\
\hline Metotrexate & $63(53.8 \%)$ \\
\hline Leflunomide & $15(12.8 \%)$ \\
\hline Sulfasalazine & $10(8.5 \%)$ \\
\hline Cyclophosphamide & $11(9.4 \%)$ \\
\hline Azathioprine & $23(19.6 \%)$ \\
\hline Biologic therapy & $48(41 \%)$ \\
\hline Corticosteroids & 46 (39.3\%) \\
\hline
\end{tabular}

\section{Immunological profile in relatives of patients with AR}

The main purpose of the present study was to determine the number of first and second-degree relatives who present serum levels of RF and anti-CCP antibodies.

The identification of the immunological profile of the individuals is a very important step for both diagnosis and identification of the high risk incidences of RA, but also for the description of certain subgroups with different progressive characteristics.

117 first- and second-degree relatives of RA patients were tested using a single blood sample, determining rheumatoid factor by immunoturbidimetry and anti-CCP antibodies using enzyme linked immunosorbent assay (ELISA) technique.

The mean age of the relatives was 43.2 years, first degree-relatives being in $70.9 \%$ of cases.

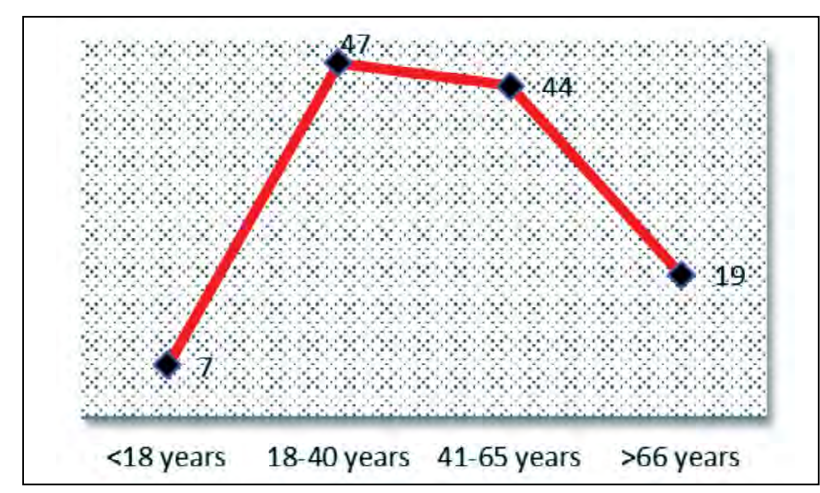

FIGURE 4. Age distribution of relatives

The evaluation of the immunological profile in the relatives found $7.69 \%$ positive percentage for anti-CCP antibodies. Multiple studies have evaluated the role and diagnostic sensitivity of anti-CCP antibodies using various tests that determine both anti-CCP1 and anti-CCP2 antibodies. The first author who reported the usefulness of anti-CCP antibodies in AR was Schelleken in 1988 (10), who have demonstrated a specificity of these antibodies up to $98 \%$.

The rheumatoid factor represents a large family of immunoglobulins with various isotypes, constituting diagnostic criteria and prognostic marker in patients with RA. RF is detected in about $60-80 \%$ of the cases with long evolution, and only in $50 \%$ of the early cases of RA (11). They can also be found in 5\% of healthy subjects.

Our data identified a percentage of $42.7 \%$ relatives (50) who are RF positive. Of the 50 relatives who registered positive values of $\mathrm{RF}$, simultaneous positivity of the anti-CCP antibodies was found at $10 \%$ of cases.

The immunological tests and the sublots of the relatives can be found in the results tables below.

TABLE 2. The immunological tests

\begin{tabular}{|l|c|c|}
\hline & RA Patients (n=117) & Relatives (n=117) \\
\hline RF + & 117 & 50 \\
\hline RF - & 0 & 67 \\
\hline Anti-CCP + & 117 & 9 \\
\hline Anti-CCP - & 0 & 108 \\
\hline
\end{tabular}

TABLE 3. The sublots of the relatives

\begin{tabular}{|l|c|}
\hline & No of relatives \\
\hline RF+, anti-CCP+ & 5 \\
\hline RF-, anti-CCP+ & 4 \\
\hline RF+, anti-CP- & 45 \\
\hline RF-, anti-CCP & 63 \\
\hline Dg RA relatives & 8 \\
\hline
\end{tabular}

$6.8 \%$ relatives satisfied the ACR criteria and were thus classified as RA. Among this relatives 
studied, 6 were seropositive RA and 2 seronegative RA (negative for RF, positive anti-CCP, strong symptoms consistent with rheumatoid arthritis).

Also, specific attention should be paid to those relatives who presented serum RF positive even in the absence of any symptom, because It is well recognised that a state of autoimmunity, in which immunological tolerance is broken, precedes the development of symptoms in the majority of patients with rheumatoid arthritis (12). These relatives who needs constantly check-ups are represented in table 4.

TABLE 4. The relatives who needs constantly check-ups

\begin{tabular}{|l|l|c|}
\hline Autoimmunity & Limits & $\begin{array}{c}\text { No of } \\
\text { relatives }\end{array}$ \\
\hline \multirow{2}{*}{ RF+ } & $(>3 \times$ upper limit of normal) & 7 \\
\cline { 2 - 3 } & $(<3 \times$ upper limit of normal) & 37 \\
\hline \multirow{2}{*}{ Anti-CCP +} & $(>3 \times$ upper limit of normal) & 0 \\
\cline { 2 - 3 } & $(<3 \times$ upper limit of normal) & 2 \\
\hline
\end{tabular}

Two totally asymptomatic relatives had positive anti-CCP antibodies, which should not be neglected because the literature data have shown that the anti-CCP antibody autoimmunity can precede the development of the disease with 10-15 years before.

Regarding daughters with positive RF, there is a statistically significant correlation between daughters with increased RF and higher HAQ score in patients with RA than patients who have daughters with negative $R F(1,37$ vs. $1.15, p=0.0067)$.

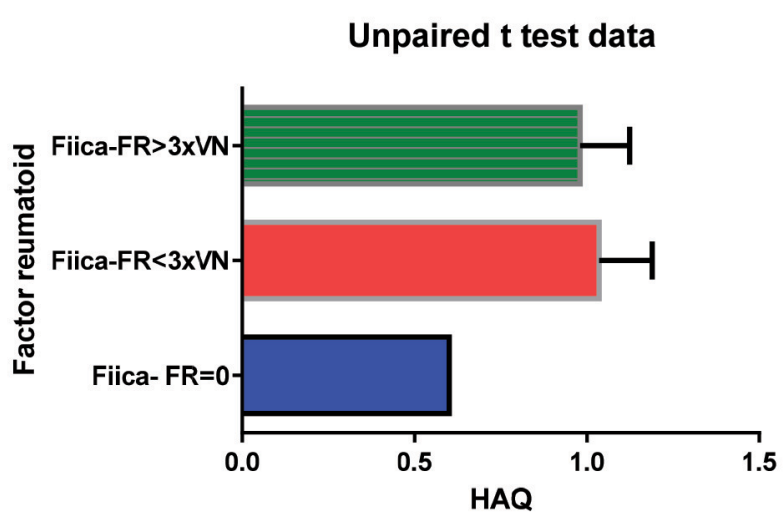

FIGURE 5. Correlation between RF positive daughters and $H A Q$ score of RA patients
There is a moderate association between smoking and the presence of RF in relatives $(\mathrm{k}=0.57)$ (,The Measurement of Interrater Agreement", n.d.), although epidemiological studies have identified smoking as an important risk factor for RA. Other previous studies provided quantitative evidence that smoking is an important risk factor only for women in developing RA (13). In our analysis there were no significant differences in female and male smokers. RF positivity was observed in 38 smoker relatives.

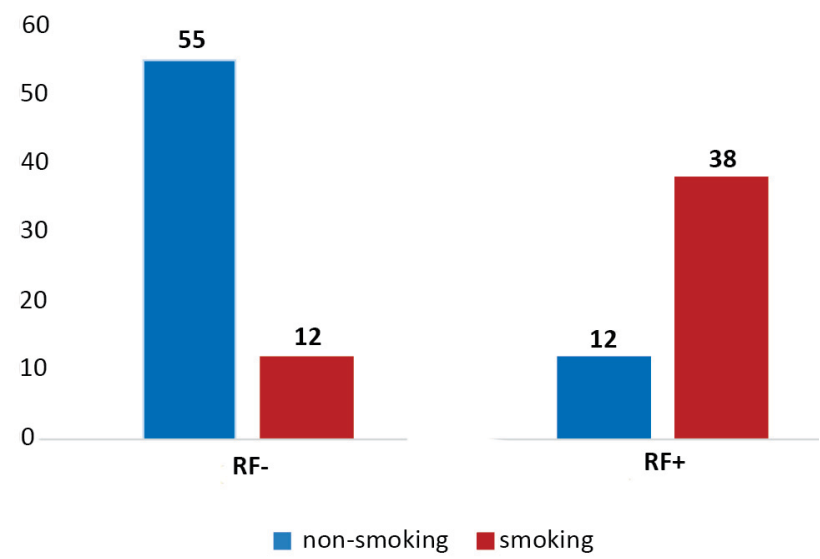

FIGURE 6. Correlation between smoking and RF positivity in relatives

\section{DISCUSSION}

The hypothesis of familial aggregation is demonstrated in the present study, especially on the the first degree-relatives in descending line, the dominant being held on maternal line ( 5 daughters presented anti-CCP antibodies).

The present study showed that first-degree relatives of patients with RA have a higher prevalence of RA autoantibodies, especially RF-positive.

The identification of the immunological profile of the patients is a very important step for both diagnosis and identification of the subjects with high risk of RA.

It can be attested that the high specific immunological profile (rheumatoid factor and anti-CCP antibodies), associated with the correctly performed anamnesis, physical exam and the paraclinical

TABLE 5. Correlation between RF in relatives and extra-articular manifestations in patients with $R A$

\begin{tabular}{|l|c|c|c|c|c|}
\hline $\begin{array}{l}\text { Extra-articular } \\
\text { manifestations }\end{array}$ & $\begin{array}{c}\text { Daughters RF + } \\
\text { (n) }\end{array}$ & $\begin{array}{c}\text { Daughters RF - } \\
\text { (n) }\end{array}$ & Relative risk & P value & $\begin{array}{c}\text { Confindence } \\
\text { interval 95\% }\end{array}$ \\
\hline Cardiac & 8 & 2 & 0.7337 & 0,2905 & $0.4950-1.088$ \\
\hline Pulmonary & 4 & 2 & 0.9300 & 1.0000 & $0.5073-1.705$ \\
\hline Skin & 4 & 4 & 1.292 & 0.4561 & $0.6261-2.665$ \\
\hline Rheumatoid nodules & 5 & 1 & 0.7200 & 0.3932 & $0.4714-1.100$ \\
\hline Sjögren's syndrome & 5 & 1 & 0.7200 & 0.3932 & $0.4714-1.100$ \\
\hline
\end{tabular}


investigations are extremely useful in identifying relatives at risk of developing RA.

$\mathrm{RF}$ and anti-CCP should be done to relatives who have family history of RA, and mandatory to those who presenting pain, swelling, or morning stiffness in their hand joints, or in other joints.

\section{CONCLUSIONS}

117 relatives of patients with RA were tested using a single blood sample, determining rheumatoid factor by immunoturbidimetric assay and anti-CCP antibodies using the ELISA technique.

67 relatives were between the ages of 18 and 40 .

The evaluation of the immunological profile in the relatives found a $7.69 \%$ positive percentage for anti-CCP antibodies. Our data identified a percentage of $42.7 \%$ relatives who were RF positive. Of the 50 relatives who registered positive values of RF, simultaneous positivity of the anti-CCP antibodies was found at $10 \%$ of cases.

$6.8 \%$ relatives satisfied the ACR criteria and were thus classified as RA. Among this relatives studied, 6 were seropositive RA and 2 seronegative $\mathrm{RA}$. The titres of anti-CCP were markedly higher for patients who satisfied the ACR criteria than those who did not. Hence a significant percentage of relatives would miss treatment for RA if this study had not been done.

There was a statistically significant correlation between daughters with increased RF with higher HAQ in patients with RA than patients with daughters seronegative (1.37 vs. $1.15, \mathrm{p}=0.0067)$.

Children with anti-CCP antibodies positive correlate with increased disease activity in patients with RA. The correlation is statistically significant $(\mathrm{p}=$ 0.0009).

We founded a moderate association between smoking and the presence of rheumatoid factor in relatives of patients in the study group $(\mathrm{k}=0.57)$.

The most important fact of this study is that 8 relatives were diagnosed accurately with RA and received the appropriate treatment.

Conflict of interest: none declared Financial support: none declared

\section{REFERENCES}

1. Yarwood A, Huizinga TW, Worthington J. The genetics of rheumatoid arthritis: risk and protection in different stages of the evolution of RA. Rheumatology (Oxford) 2016;55:199-209.

2. Somers EC, Antonsen $S$, Pedersen $L$ et al. Parental history of lupus and rheumatoid arthritis and risk in offspring in a nationwide cohort study: does sex matter? Ann Rheum Dis 2013;72:525-9.

3. Kim K, Bang SY, Lee HS et al. High-density genotyping of immune loci in Koreans and Europeans identifies eight new rheumatoid arthritis risk loci. Ann Rheum Dis 2015;74:e13 10.

4. Ferucci ED, Darrah E, Smolik I et al. Prevalence of antipeptidylarginine deiminase type 4 antibodies in rheumatoid arthritis and unaffected first-degree relatives in indigenous North American Populations. J Rheumatol 2013;40:1523-8.

5. Sparks JA, Iversen MD, Miller Kroouze R et al. Personalized Risk Estimator for Rheumatoid Arthritis (PRE-RA) Family Study: rationale and design for a randomized controlled trial evaluating rheumatoid arthritis risk education to first-degree relatives. Contemp Clin Trials 2014;39:145-57.

6. Novotny F, Haeny S, Hudelson P et al. Primary prevention of rheumatoid arthritis: A qualitative study in a high-risk population. Joint Bone Spine 2013;80:673-4.

7. Finckh A, Liang MH, van Herckenrode CM, de Pablo P. Longterm impact of early treatment on radiographic progression in rheumatoid arthritis: A meta-analysis. Arthritis Rheum. 2006 Dec 15; 55(6):864-72.

8. Smolik I, Robinson DB, Bernstein CN, El-Gabalawy HS. Firstdegree relatives of patients with rheumatoid arthritis exhibit high prevalence of joint symptoms. J Rheumatol. 2013 Jun; 40(6):818-24 .

9. Aletaha D, Neogi T, Silman AJ, Funovits J, Felson DT, Bingham $\mathrm{CO}$, Hawker G. Rheumatoid arthritis classification criteria: An American College of Rheumatology/European League Against Rheumatism collaborative initiative. Arthritis \& Rheumatism 2010 , 62(9), 2569-2581.

10. Schellekens $G A$, de Jong $B A$, van den Hoogen FH, van de Putte LB, van Venrooij WJ. Citrulline is an essential constituent of antigenic determinants recognized by rheumatoid arthritis-specific autoantibodies. The Journal of Clinical Investigation 1998, 101(1), 273-281.

11. Chou C, Liao H, Chen Ch, Chen W, Wang H, Su K. The Clinical Application of Anti-CCP in Rheumatoid Arthritis and Other Rheumatic Diseases. Biomark Insights. 2007;2:165-171.

12. Tracy A, Buckley CD, Raza K. Pre-symptomatic autoimmunity in rheumatoid arthritis: When does the disease start? Semin Immunopathol. 2017; 39(4):423-435.

13. Chang K, Yang SM, Kim SH, Han KH, Park SJ, Shin JI. Smoking and rheumatoid arthritis. Int J Mol Sci. 2014; 15(12):22279-22295. 